\title{
A Compact Triple-Band Negative Permittivity Metamaterial for C, X-Band Applications
}

\author{
Dushyant Marathe and Kishore Kulat \\ Department of Electronics and Communication Engineering, Visvesvaraya National Institute of Technology (VNIT), \\ South Ambazari Road, Nagpur, India \\ Correspondence should be addressed to Dushyant Marathe; dushyantmarathe@gmail.com
}

Received 6 July 2017; Accepted 18 September 2017; Published 18 October 2017

Academic Editor: Ikmo Park

Copyright (C) 2017 Dushyant Marathe and Kishore Kulat. This is an open access article distributed under the Creative Commons Attribution License, which permits unrestricted use, distribution, and reproduction in any medium, provided the original work is properly cited.

\begin{abstract}
We report a new design of triple-band electric metamaterial resonator (TBEMR) based on integration of open delta loops within square ring resonator. This metamaterial resonator has three distinct $\varepsilon$-negative regions (ENG) over $\mathrm{C}$, X frequency bands. The transmission and reflection response of the proposed subwavelength resonator is analyzed using full-wave electromagnetic solver Ansys HFSS to demonstrate the presence of electrical resonances within frequencies $4-12 \mathrm{GHz}$. Effective medium parameters permittivity and permeability are extracted from simulated complex scattering parameters to verify existence of ENG regions. The investigations are also carried out regarding dependence of resonant frequencies on design parameters of the TBEMR unit cell. The effective medium ratio $\left(\lambda_{0} / a\right)$ for proposed subwavelength resonator is compared with various other metamaterial resonators to indicate its compact nature.
\end{abstract}

\section{Introduction}

Metamaterials are artificially structured composites made of subwavelength resonators that exhibit unique electromagnetic properties not found in individual constituent components. The classification of metamaterials is carried as $\varepsilon$-negative (ENG), $\mu$-negative (MNG), or double-negative (DNG) based on negative values for either permittivity, permeability, or both [1]. These single negative metamaterials including ENG and MNG have exotic electromagnetic properties different from most naturally occurring materials. The first artificial realization of metamaterial was made of two different types of subwavelength structures, thin wire and split ring resonator (SRR), to get negative response for permittivity and permeability over common frequency range [2]. Metamaterials with negative refractive index can be realized by combining the single negative ENG and MNG structures within same unit cell [3].

These single negative metamaterials are important constituent in the formation of negative index LH metamaterials. Additionally, these single negative metamaterials also find applications in gain enhancement for antennas [4], microwave absorber [5], material characterization [6], and microwave sensors $[7,8]$. Applications in microwave devises such as gain enhancement or beam forming for antennas for multiband operations require multiband characteristics for metamaterials [9]. To solve these problems several single negative (SNG) metamaterial structures having multiband responses were reported in the literature [10-13]. A butterfly pattern resonator reported in [10] has triple-band response with two magnetic and one electric resonance. A four-arm square spiral metamaterial reported in [11] has dual-band magnetic and electric response in different frequency band. A square single loop resonator (square-SLR) [14] is reported to have triple-band response in X-band. This square-SLR has two distinct MNG regions and one very weak ENG region. A hybrid-SLR is reported to have quad-band response in S,C bands with two MNG and two ENG responses [12]. However, the above reported metamaterials have mixed response with MNG and ENG regions in nonoverlapping frequency bands. Conversely, the nested $\mathrm{U}$-shaped ring resonators reported in [13] have triple-band response with only MNG characteristics 
in X-band region. A summary on conventional ENG metamaterials and recent developments in it are reviewed in the following paragraphs.

Conventional, single negative ENG metamaterials like thin wire structure were shown to have dielectric function to be of the form like plasma [15]. A planar complementary SRR reported in [16] shows ENG response and acts as an alternative to thin wire structure. However, thin wire and CSRR structure due to their interplane connectivity impose difficulty in fabricating countered objects like lenses. As a shortfall of this, Schurig et al. [17] proposed electrically coupled LC resonator for obtaining ENG response. Prime advantages of E-LC resonator are planar design, pure-electric response due to mirror symmetry, no need of intercell and interplane connectivity, and improved $\lambda_{0} / a$ ratio. The E-LC resonator was miniaturized further by replacing capacitive gap with interdigitated (IDC) capacitance [18]. The maximum value for effective medium ratio $\left(\lambda_{0} / a\right)$ achieved was 10.2 . It has advantage of improved homogeneity and electrical size reduction without any fabrication limitations. However, these advantages were achieved at the cost of reduced coupling to the external field as indicated by low value $(-15 \mathrm{~dB})$ of transmission minima. Kolb et al. [19] proposed other notable low resonant frequency structures derived from E-LC resonator for further improvement in $\lambda_{0} / a$ ratio. These structures were inspired from original E-LC resonator. It includes geometries such as I-shaped resonator, thin square loop, meandershaped resonator, and its variations. A meander-shaped resonator presents electrical size reduction with lowest resonant frequency. However, it occurs at the cost of cross-polarization effect and reduced coupling to the external field. A planar Zshaped resonator [20] for negative permittivity was shown to have lower resonant frequency than conventional E-LC resonator with same physical size. The double S-shaped resonators realized in $[21,22]$ give overlapped response for negative permittivity and permeability. However, above reported metamaterial resonators were having single-band response for negative permittivity property.

In recent years, research in this particular area includes electrical size reduction, improvement in effective medium ratio [23], and achieving dual-band characteristics [24-26]. Size reduction is achieved by a common idea of raising overall inductance and capacitance in the unit cell, thus achieving lower resonant frequency. The approaches include changing geometrical feature dimensions in the structure, that is, shortening the gaps or lengthening the metallic strips [23], and use of surface mounted capacitor [27] and high permittivity substrate as host material [25]. However, above approaches have limitations due to difficulty and complexity in the fabrication and cost and availability of high permittivity substrates. The dual-band characteristic is obtained by combining different resonating structures and optimizing them to get response in desired frequency band [24-26]. A pair of E-LCs resonators with different sized inductive loops and capacitive gaps were shown to have two distinct electric resonances [24, 25]. However, shortfalls in these designs are poor coupling to external field at higher resonant frequency [24] and use of high permittivity material as substrate [25]. A dualband negative permittivity metamaterial design based on integration of the delta loop within closed square ring resonator was proposed in [26]. However, meander loaded delta increases complexity of the design.

Metamaterial design proposed in this paper has purely epsilon-negative $(\varepsilon<0)$ response with triple-band characteristics. The multiband responses for this structure are obtained by integrating open delta loops with square ring resonator. Moreover effect of design parameters such as arm length of delta loops, position of shorting stubs, gaps formed due to open delta loops, metallic strip width, and substrate properties are investigated in detail. Also, proposed resonator shows notable improvements over other multiband structures in terms of pure-electric response, polarization stable response, use of low cost FR-4 as host material, and design simplicity for ease in the fabrication at higher frequencies.

\section{Design and Simulations of Triple-Band Electric Metamaterial Resonator (TBEMR)}

In this communication, triple-band electric metamaterial resonator (TBEMR) is proposed and investigated which couples strongly to electric component of the incident field. Design of this metamaterial resonator is based on integrating open delta loops within square ring resonator. The geometry of proposed resonator is described in Figure 1 together with design parameters and incident electromagnetic wave excitation. We started with square loop resonator that already appeared in the literature [19]. The square loop resonator that couples strongly to electric component of electromagnetic wave is widely used for getting $\varepsilon$-negative response for normal wave incidence as shown in $[19,29]$. Although square loop resonator has advantage in terms of polarization insensitive behaviour for normally incident wave [29], the disadvantage is regarding single-band response and electrically large size as indicated by very high value of $\lambda_{0} / a$. The open delta loop resonator (ODLR) proposed by Sabah [30] is shown to have single-band magnetic response for parallelly incident wave, whereas Sarkhel [26] has recently shown electric response for meander-line loaded closed delta loop resonator (CDLR) for normally incident wave. However disadvantage of both designs is that they are highly polarization sensitive. In case of our design reported as shown in Figure 1(a), we have used open delta loop resonator (ODLR) formed by metallic strips $l_{s 1}, l_{x 2}, l_{y 2}$, and $l_{s 2}$. Two such ODLRs are used in the design of TBEMR. The open vertex of the each ODLR is connected with the diagonally opposite end of square ring resonator by shorting stubs $s_{1}$ and $s_{2}$. The gaps formed between the base portions of the adjacent ODLR are responsible for creating capacitances. Due to diagonal symmetry of the structure, we get exactly same triple-band response for vertically ( $y$-polarized) and horizontally ( $x$-polarized) polarized electromagnetic wave when structure is rotated by angle $90^{\circ}$ around $z$-axis.

The proposed TBEMR is to be printed on single face copper cladded FR-4 epoxy dielectric spacer having permittivity $\varepsilon_{r}=4.4$, loss tangent $(\tan \delta)$ of 0.02 , and a thickness of $1.6 \mathrm{~mm}$. The unit cell of the proposed resonator has physical dimensions of $6 \mathrm{~mm} \times 6 \mathrm{~mm}$. All metallic strips in the design 


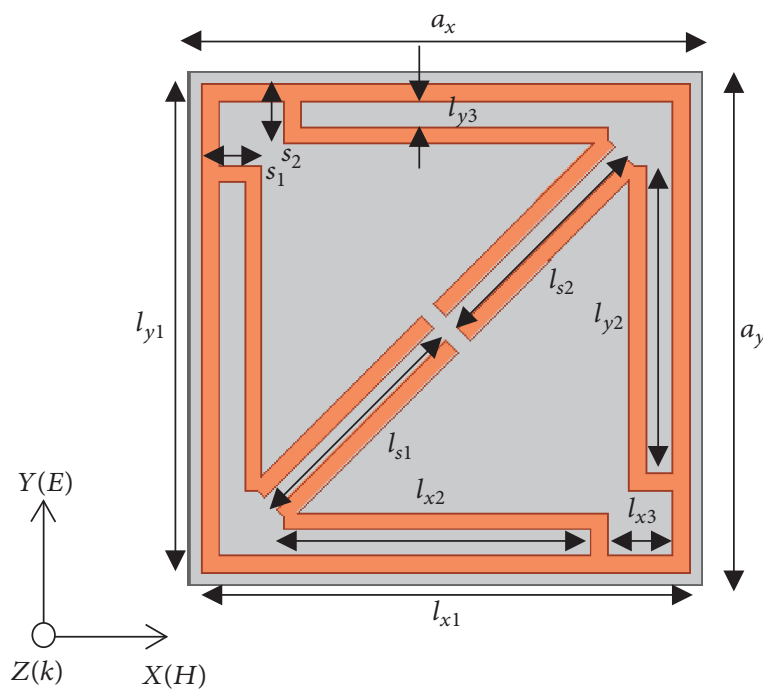

(a) TBEMR unit cell

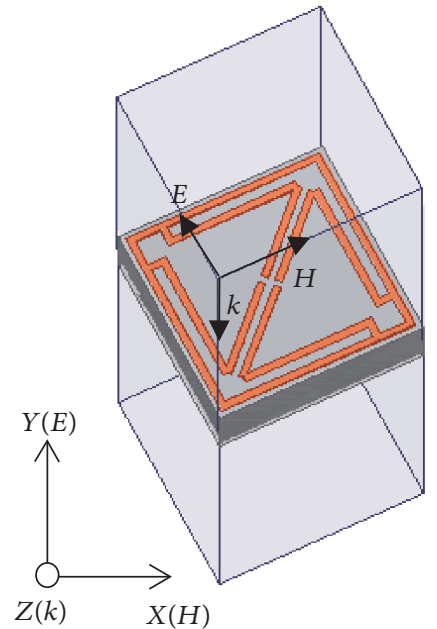

(b) Simulation setup

FIgURE 1: (a) Geometry. (b) Simulation setup for TBEMR unit cell using PEC-PMC boundaries. For the proposed resonator geometrical parameters are $a_{x}=6 \mathrm{~mm}, a_{y}=6 \mathrm{~mm}, l_{x 1}=l_{y 1}=5.7 \mathrm{~mm}, l_{x 2}=l_{y 2}=3.6 \mathrm{~mm}, l_{x 3}=0.75 \mathrm{~mm}, l_{y 3}=0.3 \mathrm{~mm}$, and $l_{s 1}=l_{s 2}=2.8 \mathrm{~mm}$. The lattice constant of the unit cell is $6 \mathrm{~mm} \times 6 \mathrm{~mm}$ and width of all metallic strips is $w=0.2 \mathrm{~mm}$.

of TBEMR are made of copper having conductivity of $5.8 \times$ $10^{7} \mathrm{~S} / \mathrm{m}$ and thickness of $35 \mu \mathrm{m}$. The copper line width considered in simulation is $0.2 \mathrm{~mm}$ for fabrication tolerances. The electromagnetic response from the proposed resonator is investigated using finite element method based Ansys HFSS solver version 14. Simulations are performed on a unit cell of proposed resonator by applying perfect electric (PEC) and perfect magnetic (PMC) boundary conditions. The PEC and PMC boundaries along with two wave ports model source of plane wave excitation and indicate that unit cells are repeated infinitely along $X$ and $Y$ direction. Perfect electric and perfect magnetic boundaries are assigned to the planes parallel to $X Z$ and $Y Z$. This indicates that electric field is oriented along $y$ axis and magnetic field is oriented along $x$-axis as depicted in Figure 1(b). We obtained complex scattering parameters $S_{11}$ and $S_{21}$ from unit cell simulation in the frequency range of 3-12 GHz. The complex S-matrix data is deembedded appropriately at the edges of the cubic unit cell. At a higher value of frequency sweep, that is, $12 \mathrm{GHz}$, the ratio $\lambda_{0} / a$ has value 4.16 which is in accordance with the limit of 4 . This ensures that unit cell dimensions are much smaller than operating wavelength and it conforms to the effective medium theory without introducing undesired effects of spatial dispersion [31].

Numerically simulated magnitude and phase of transmission $\left(S_{21}\right)$ and reflection $\left(S_{11}\right)$ spectra against frequency for proposed TBEMR is presented as shown in Figures 2(a) and 2(b). The dip in transmission spectra $S_{21}$ indicates stopband response and is an indication of resonance for single negative metamaterial structure. The transmission minima $\left(S_{21}\right)$ is displayed at resonant frequencies $4.32 \mathrm{GHz}, 7.55 \mathrm{GHz}$, and 9.76 GHz as shown in Table 1. This fact is also confirmed from the pulse like transition seen in transmission phase spectra at respective resonant frequencies as shown in Figure 2(b). The
TABLE 1: Simulated resonant frequency $f_{0}(\mathrm{GHz})$, transmission minima $S_{21}(\mathrm{~dB})$, and effective medium ratio $\left(\lambda_{0} / a\right)$.

\begin{tabular}{lccc}
\hline Meta-atom & $f_{0}(\mathrm{GHz})$ & $S_{21}(\mathrm{~dB})$ & $\left(\lambda_{0} / a\right)$ \\
\hline TBEMR & $4.32,7.55,9.76$ & $-23.29,-34.32,-34.76$ & $11.5,6.6,5.1$ \\
\hline
\end{tabular}

absorbance spectra for proposed resonator can be calculated from expression $A(f)=1-\left|S_{11}(f)\right|^{2}-\left|S_{21}(f)\right|^{2}$ as shown in Figure 3. It peaks at frequencies $4.65 \mathrm{GHz}$ and $8.38 \mathrm{GHz}$, where reflection spectra has minimum value. The absorptivity has maximum value of 0.36 and 0.31 for proposed resonator and it can be minimized to some extent by using high conductivity metal and high quality substrate. Although loss characteristic is drawback for many applications but it can be turned into an advantage for application like wave absorber [32]. The ideal value for loss characteristics $(A(f))$ is near unity. It can be achieved by matching surface impedance of metamaterial slab with wave impedance and minimizing wave transmission in manner as shown in [32].

\section{Parameter Extractions, Surface Current, and Electric Field Distribution}

The effective medium parameters are extracted from the simulated complex reflection and transmission coefficients of wave incident on the metamaterial unit cell. A computer program is written to retrieve effective impedance, refractive index, effective permittivity, and permeability based on the formulation that appeared in [33]. For normally incident plane wave on the slab of a metamaterial unit cell, constitutive parameters such as effective impedance and refractive index 


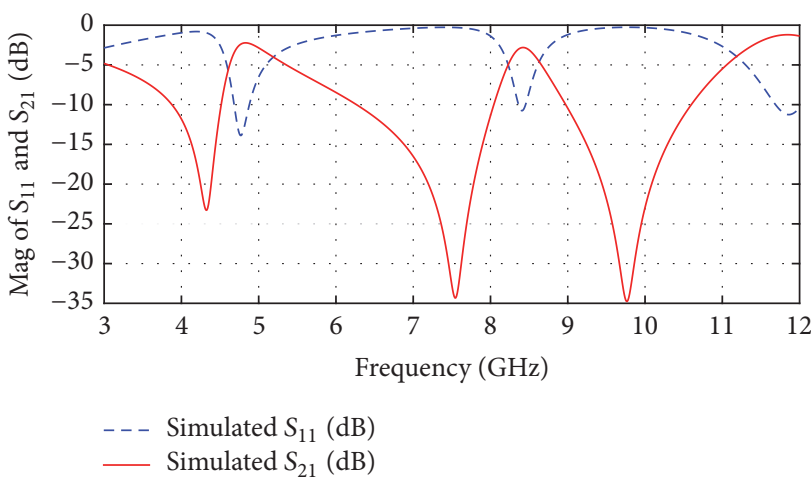

(a)

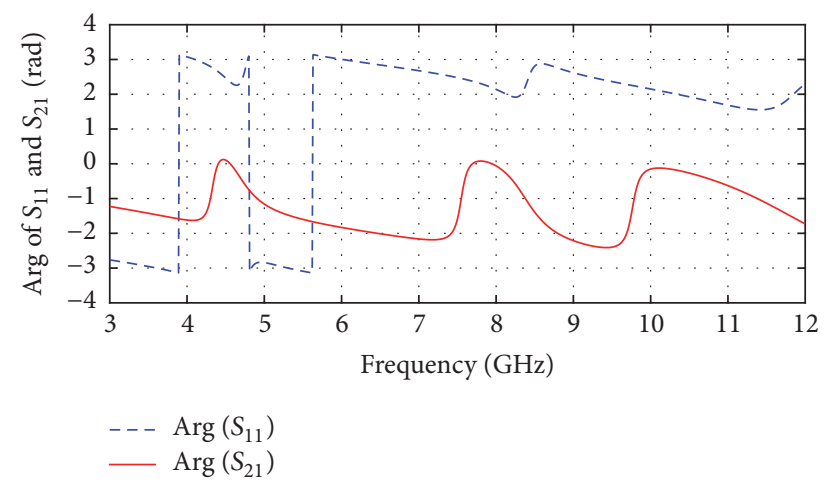

(b)

Figure 2: Simulated transmission and reflection characteristics: (a) magnitude spectra of $S_{11}$ and $S_{21}$, (b) phase spectra of $S_{11}$ and $S_{21}$.

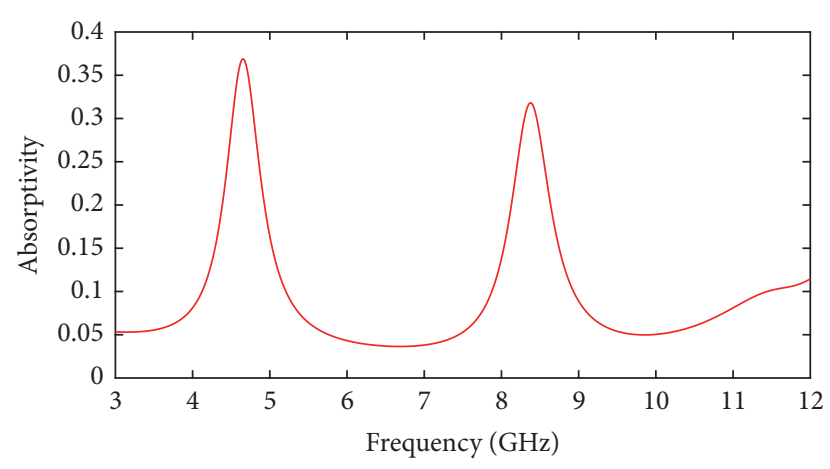

FIGURE 3: Absorption spectra $A(f)$ for proposed triple-band electric metamaterial resonator (TBEMR).

can be computed from Fresnel's relation by inverting S-matrix data.

$$
z_{\text {eff }}= \pm \sqrt{\frac{\left(1+S_{11}\right)^{2}-S_{21}^{2}}{\left(1-S_{11}\right)^{2}-S_{21}^{2}}} .
$$

The sign of the wave impedance $z_{\text {eff }}$ in (1) is determined by imposing the conditions of passive material, that is, real part of the effective impedance $\operatorname{Re}\left(z_{\text {eff }}\right)$ and imaginary part of refractive index $\operatorname{Im}\left(n_{\text {eff }}\right)$, to be greater than zero.

$$
e^{\mathrm{in}_{\mathrm{eff}} k_{0} d_{\mathrm{eff}}}=\frac{S_{21}}{1-S_{11} R_{01}} .
$$

The complex refractive index can be determined from

$$
\begin{aligned}
n_{\mathrm{eff}} & =\frac{1}{k_{0} d_{\mathrm{eff}}}\left\{\operatorname{Im}\left[\ln \left(e^{\mathrm{in} \mathrm{eff}_{0} k_{\mathrm{eff}}}\right)\right]+2 m \pi\right. \\
& \left.-i \operatorname{Re}\left[\ln \left(e^{\mathrm{in} \mathrm{e}_{\mathrm{eff}} k_{0} d_{\mathrm{eff}}}\right)\right]\right\} \quad m=0,1,2 \ldots,
\end{aligned}
$$

where $m$ is an integer denoting the branch index of complex logarithm, $d_{\text {eff }}$ is the thickness of the unit cell of metamaterial sample, $k_{0}$ is the wavenumber, and $R_{01}=\left(z_{\text {eff }}-1\right) /\left(z_{\text {eff }}+1\right)$ depends on wave impedance $z_{\text {eff }}$. The imaginary part of $n_{\text {eff }}$ in (3) is not affected by the branches of logarithm function and it can be determined accurately. However, real part has multiple solutions due to branch ambiguity. It can be determined by the method based on the Kramers-Kronig relations to ensure the uniqueness of the effective parameters [34]. The effective permittivity and permeability can be determined from the effective impedance $z_{\text {eff }}$ and refractive index $z_{\text {eff }}$ as shown below

$$
\begin{aligned}
& \varepsilon_{\text {eff }}=\frac{n_{\text {eff }}}{z_{\text {eff }}} \\
& \mu_{\text {eff }}=n_{\text {eff }} z_{\text {eff }} .
\end{aligned}
$$

The effective medium parameters permittivity, permeability, and refractive index are obtained using the above formulation for the proposed TBEMR metamaterial resonators as shown in Figures 4(a), 4(b), and 4(c), respectively. For triple-band metamaterial (TBEMR), the electric resonance is clearly seen from the real part of permittivity which varies from high positive to negative values near narrow range around resonant frequency as shown in Figure 4(a). The real part of permittivity has negative values over frequencies 4.24-4.5 GHz, $6.6-8.28 \mathrm{GHz}$, and $8.95-11.28 \mathrm{GHz}$ for first, second, and third band respectively. The proposed TBEMR shows only electric response and no magnetic response as observed from Figures 4(a) and 4(b). The plot of effective permeability has near unity value. However, antiresonant response is observed in corresponding negative permittivity frequency band. The antiresonant behaviour is an intrinsic property of such resonators due to finite spatial periodicity [35]. The antiresonant response is also seen from the imaginary part of the effective permeability which is negative at resonant frequency showing unphysical behaviour.

In quasistatic limit, when size of the resonator is very small compared to operating wavelength, it can be approximated with the LC resonant circuit. The resonant frequency is inversely proportional to square root of inductance and capacitance $\left(f_{0}=1 / 2 \pi \sqrt{L_{e} C_{e}}\right)$. The current induced in the metallic strips offers inductance, whereas gaps within the unit cell or edges between adjacent unit cells form capacitance. Thus, inductance and capacitance are influenced mainly by geometrical design parameters of the unit cell. The surface current density and electric field distributions can be plotted 


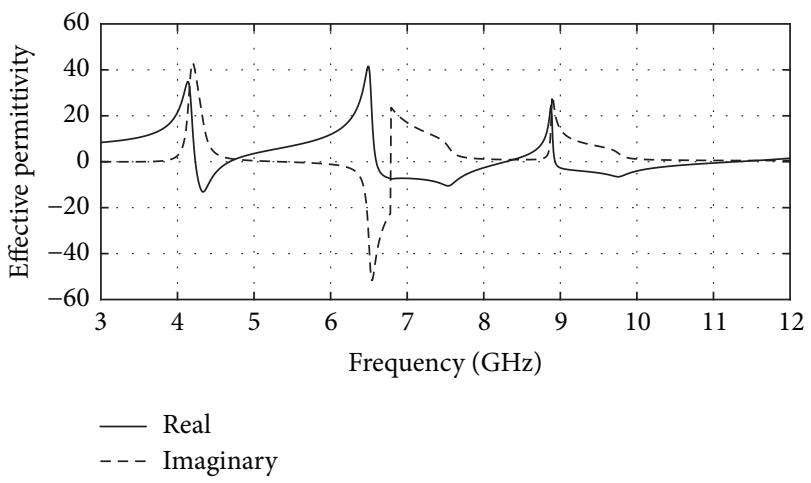

(a)

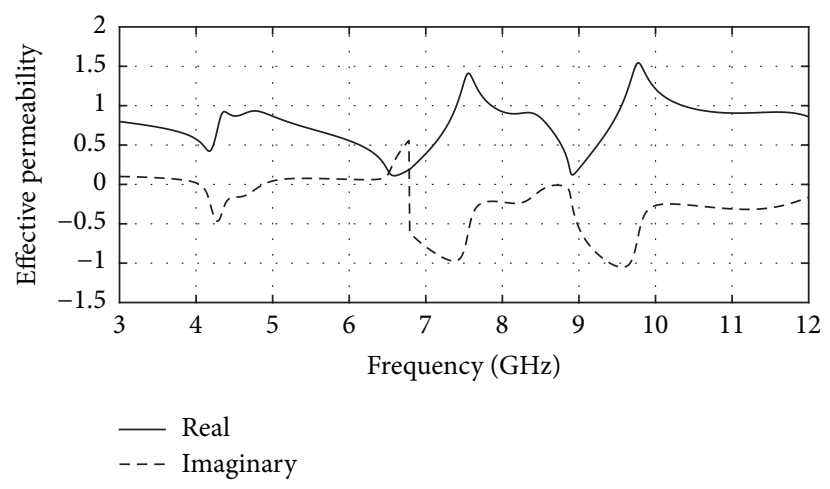

(b)

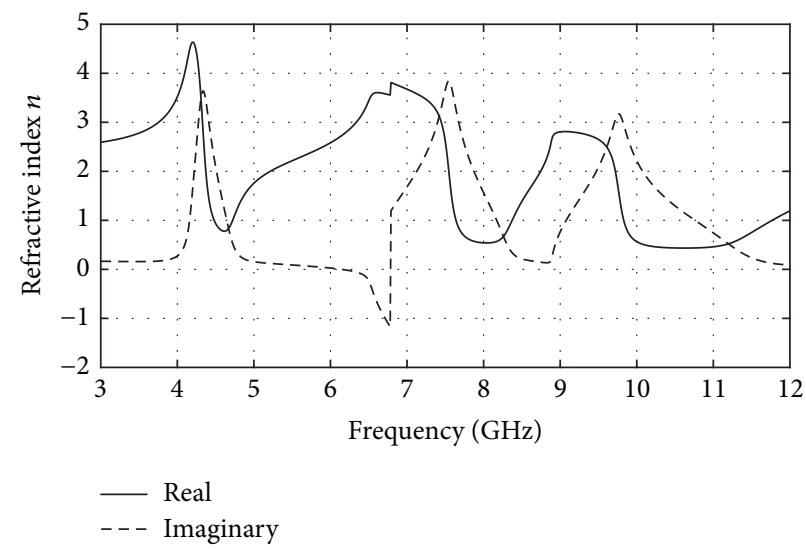

(c)

FIGURE 4: Computed effective medium parameters for the proposed triple-band electric metamaterial resonator (TBEMR): (a) permittivity, (b) permeability, and (c) refractive index, respectively. The solid lines represent the real part while dashed line represents imaginary part.

at respective resonant frequencies to understand inductive and capacitive response from the structure (refer to Figures 5 and 6, resp.). At lower resonant frequency, that is, $f_{01}=$ $4.32 \mathrm{GHz}$, it is observed that the surface current flows over top right and bottom left portion of the TBEMR resonator (refer to Figure 5(a)). The surface current flows in clockwise and anticlockwise manner in arrow-like portion of the resonator. This, clockwise and anticlockwise flow of the time varying surface current in the upper right and lower left metallic portion of the resonator cancels any magnetic dipolar activity along $z$-axis near the resonance. The magnetic field from the external plane wave excitation is oriented along $x$-axis and it lies in the plane $X-Y$ of substrate without threading the metallic loops of the TBEMR resonator. This fact explains that resonator does not couple to magnetic field component of the plane wave electromagnetic excitation. The strong intensity of the localized electric field observed near upper right and lower left inclined strips of the unit cell as shown in Figure 6(a) indicates capacitive effects. The two delta loops are coupled by mean of capacitance strongly along inclined strips $l_{s}$. Capacitive effect occurs due to spacing formed between inclined metallic strips of delta loops as shown in Figure 6(a). The metallic strips printed on substrate are planar and not fully $3 \mathrm{D}$. The accurate expressions for capacitances for coupled parallel strips deposited on dielectric substrate can be found from reference $[36,37]$.
The equivalent circuit is drawn at resonant frequencies from the plots of surface currents and electric field intensities (refer to Figure 7). The equivalent circuit presents qualitative response from the structure and helps to understand resonance mechanism at respective resonant frequencies. The application of vertically polarized plane wave induces voltages across parallel branches of the resonator and forms capacitances. The capacitor discharges and current flows via inductive metal path along the length of arrow-like geometrical portion as shown in Figure 7(a). The self-inductance of each metal strip is calculated by using (5) from the path where current flows [36].

$$
L(\mathrm{nH})=2 \times 10^{-4} l\left[\ln \left(\frac{l}{w+t}\right)+1.193+\frac{w+t}{l}\right] .
$$

The terms $w, t$, and $l$ are strip width, strip thickness, and strip length, respectively, in microns. The mutual inductance between strips is calculated by using

$$
\begin{gathered}
M(\mathrm{nH})=2 \times 10^{-4} l_{e}\left[\ln \left\{\frac{l_{e}}{d}+\left(1+\frac{l_{e}^{2}}{d^{2}}\right)^{1 / 2}\right\}\right. \\
\left.-\left(1+\frac{d^{2}}{l_{e}^{2}}\right)^{1 / 2}+\frac{d}{l_{e}}\right],
\end{gathered}
$$




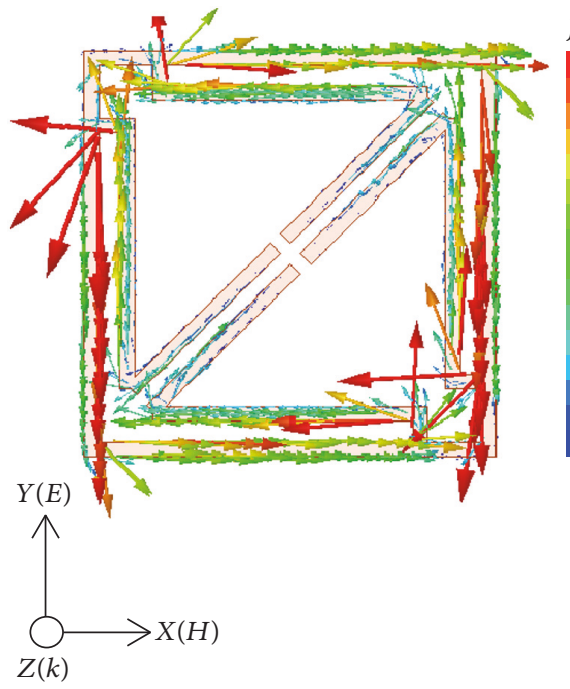

(a)
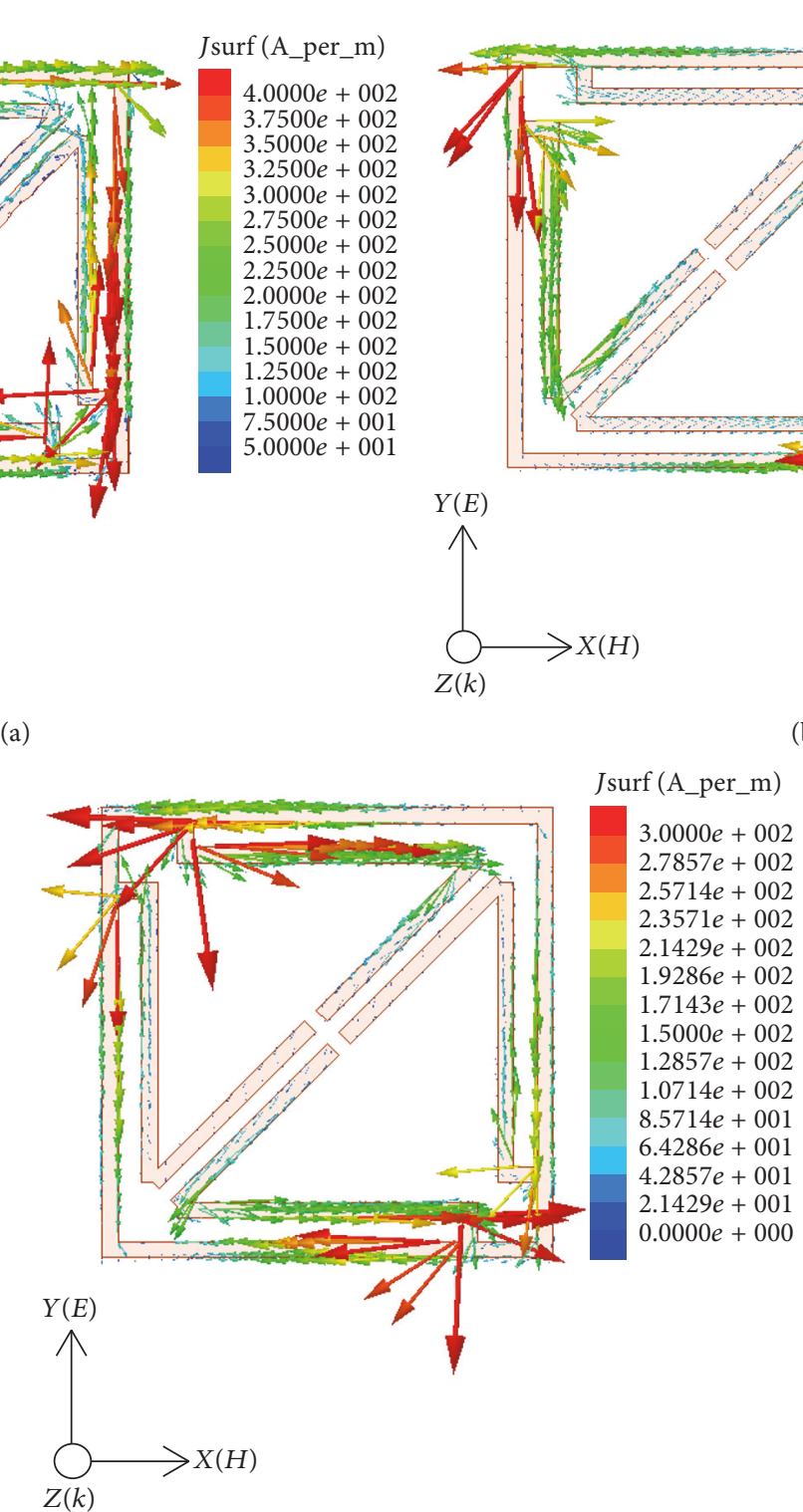

(c)

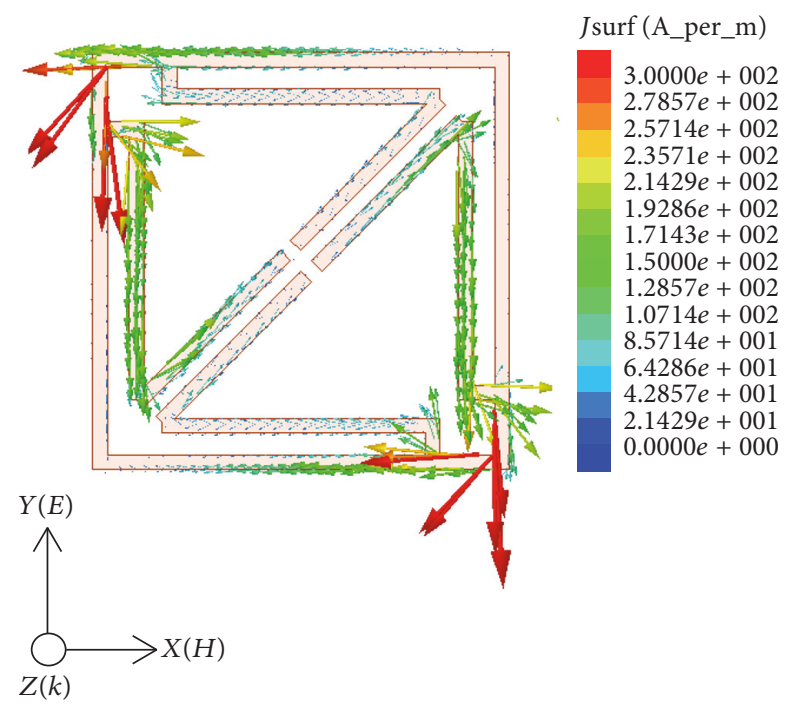

(b)

FIGURE 5: Surface current density $J_{s}$ on the metallic patterns of TBEMR metamaterial at resonant frequencies (a) $f_{01}=4.32 \mathrm{GHz},(\mathrm{b}) f_{02}=$ $7.55 \mathrm{GHz}$, and (c) $f_{03}=9.76$. Note that red color indicates strong positive values.

where $l_{e}$ is the effective length of two metal strips and $d$ is the mean distance between the strips. Dimensions $w, t, l, l_{e}$, and $d$ are in microns. The capacitance between coplanar strips is calculated by using the following $[36,37]$ :

$$
C=\varepsilon_{o} \varepsilon_{\text {eff }} \frac{K\left(k_{o}^{\prime}\right)}{K\left(k_{o}\right)},
$$

where effective dielectric constant and filling factor is defined as follows: $\varepsilon_{\text {eff }}=1+(\varepsilon-1) q, q=(1 / 2)\left(K\left(k^{\prime}\right) / K(k)\right)\left(K\left(k_{o}\right) /\right.$ $\left.K\left(k_{o}^{\prime}\right)\right), k_{o}=\sinh (\pi a / 2 h) / \sinh (\pi b / 2 h), k_{o}^{\prime}=\sqrt{1-k_{o}^{2}}, k=$ $a / b, k^{\prime}=\sqrt{1-k^{2}}, a=s / 2, b=(s / 2)+w$, where $K$ represents a complete elliptical function of first kind with $K^{\prime}$ being its complementary function [36]. $w$ and $s$ are width and spacing between coplanar metal strips. The computation of resonant frequency requires calculation of total inductance and capacitance in the structure at respective resonant mode. At first resonant frequency, that is, $f_{01}=4.32 \mathrm{GHz}$, selfinductance $\left(L_{o 1}\right)$ for the current path as shown in Figure 7(a) has value of $18.5 \mathrm{nH}$ calculated using (5). Mutual inductance $M_{1}$ between the metal strips having antiparallel current has value of $1.5 \mathrm{nH}$ calculated using (6). Then, the total inductance is calculated as $L_{e 1}=L_{o 1}-2 M_{1}=15.5 \mathrm{nH}$. The capacitance between coupled parallel strips $l_{s}$ is $C_{1}=0.1 \mathrm{pF}$. The resonant frequency is calculated as $f_{01}=1 / 2 \pi \sqrt{L_{e 1} C_{1}}=$ $4.05 \mathrm{GHz}$ which is very much close to simulated resonant frequency of $4.32 \mathrm{GHz}$. It is observed that, at this frequency, the effect of capacitor $C_{1}$ is dominant compared to intercell 


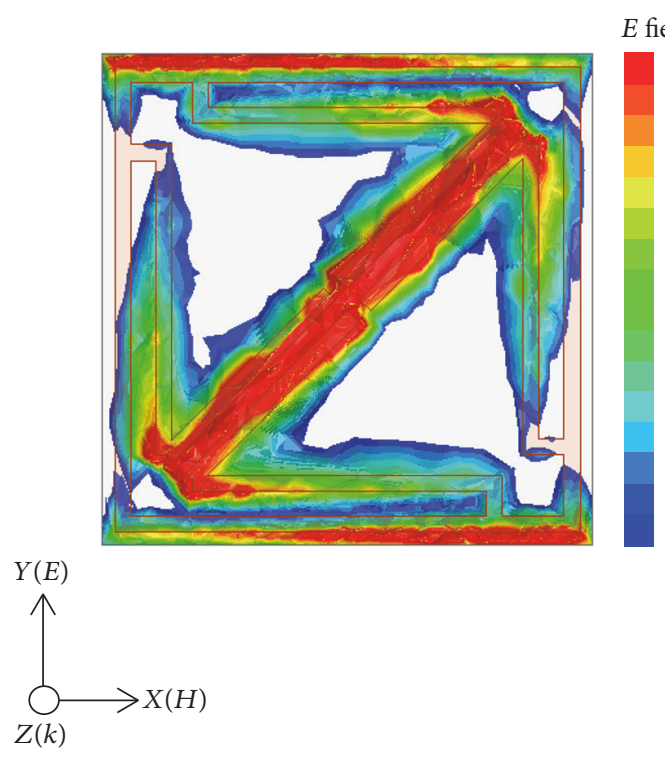

(a)
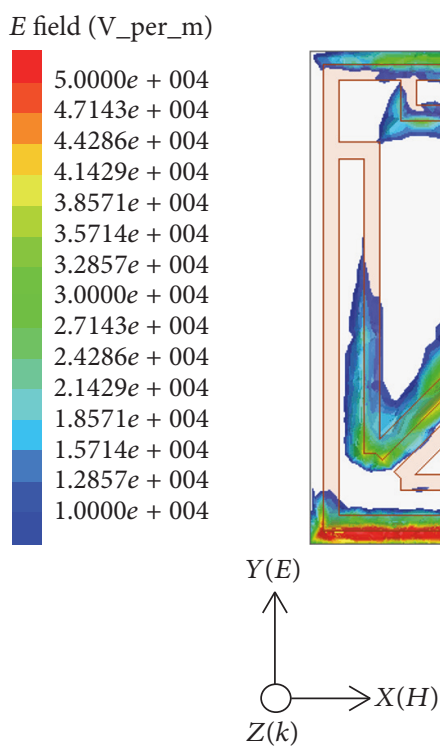

$E$ field (V_per_m)

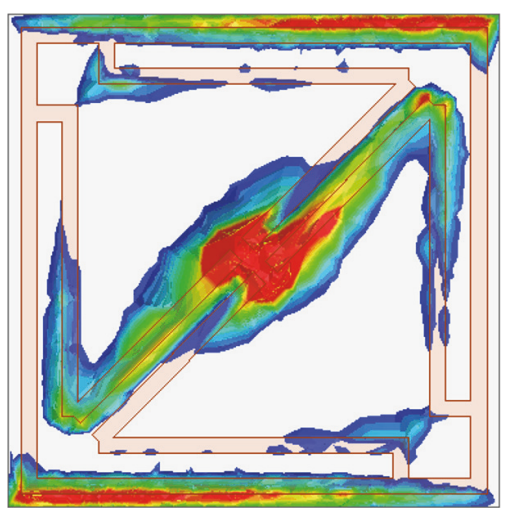

$4.0000 e+004$

$3.7857 e+004$

$3.5714 e+004$

$3.3571 e+004$

$3.1429 e+004$

$2.9286 e+004$

$2.7143 e+004$

$2.5000 e+004$

$2.2857 e+004$

$2.0714 e+004$

$1.8571 e+004$

$1.6429 e+004$

$1.4286 e+004$

$1.2143 e+004$

$1.0000 e+004$

(b)

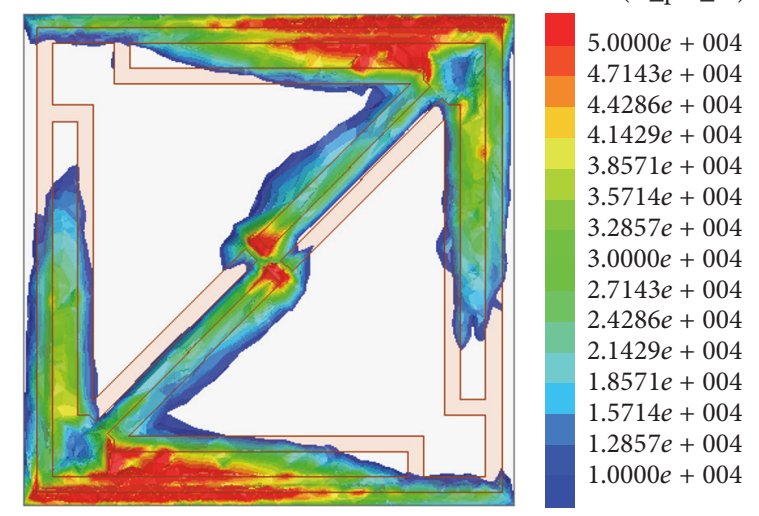

$$
\begin{gathered}
E \text { field (V_per_m) } \\
5.0000 e+004 \\
4.7143 e+004 \\
4.4286 e+004 \\
4.1429 e+004 \\
3.8571 e+004 \\
3.5714 e+004 \\
3.2857 e+004 \\
3.0000 e+004 \\
2.7143 e+004 \\
2.4286 e+004 \\
2.1429 e+004 \\
1.8571 e+004 \\
1.5714 e+004 \\
1.2857 e+004 \\
1.0000 e+004
\end{gathered}
$$

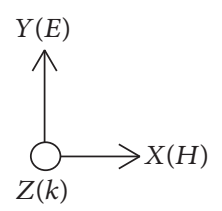

(c)

FIGURE 6: Magnitude of electric field distribution on the TBEMR metamaterial at resonant frequencies (a) $f_{01}=4.32 \mathrm{GHz}$, (b) $f_{02}=7.55 \mathrm{GHz}$, and $f_{03}=9.76$, respectively. Note that red color indicates strong positive values.

capacitance $C_{p}$. This fact is verified by observing the effect of closing the gaps and variations in gap length over resonant frequency $f_{01}$ (refer to Figures 10 and 8). Note that we have neglected ohmic losses occurring due to finite conductivity of metal strips.

At second resonant frequency, that is, $f_{02}=7.55 \mathrm{GHz}$, surface current is excited mainly along top and bottom horizontal strips of outer square loop resonator, left and right portions of delta loops as shown by arrows in Figure 7(b). The total inductance for the current path is $L_{e 2}=30.5 \mathrm{nH}$ calculated from (5). The localized electric fields can be observed at the top right and bottom left horizontal branches of the square loop. These electric fields are due to capacitances formed between gaps of the adjacent unit cells denoted as intercell capacitance $C_{p}$. The capacitance is also formed at the center within the unit cell $C_{2}$. This capacitance is relatively weak compared to that of intercell capacitance $C_{p}(0.18 \mathrm{pF})$ and has pronounced effect on determining resonant frequency as they act in series. This fact is substantially similar to that of $Z_{\text {gap }}$ resonator discussed in [20]. Determining the value of capacitor $C_{2}$ is difficult as it is formed between nonadjacent diagonally opposite arms of delta loop. However, in calculation of $f_{02}$, it is approximated as $1 / 6$ th the value of $C_{1}$. The equivalent capacitance is then calculated as 

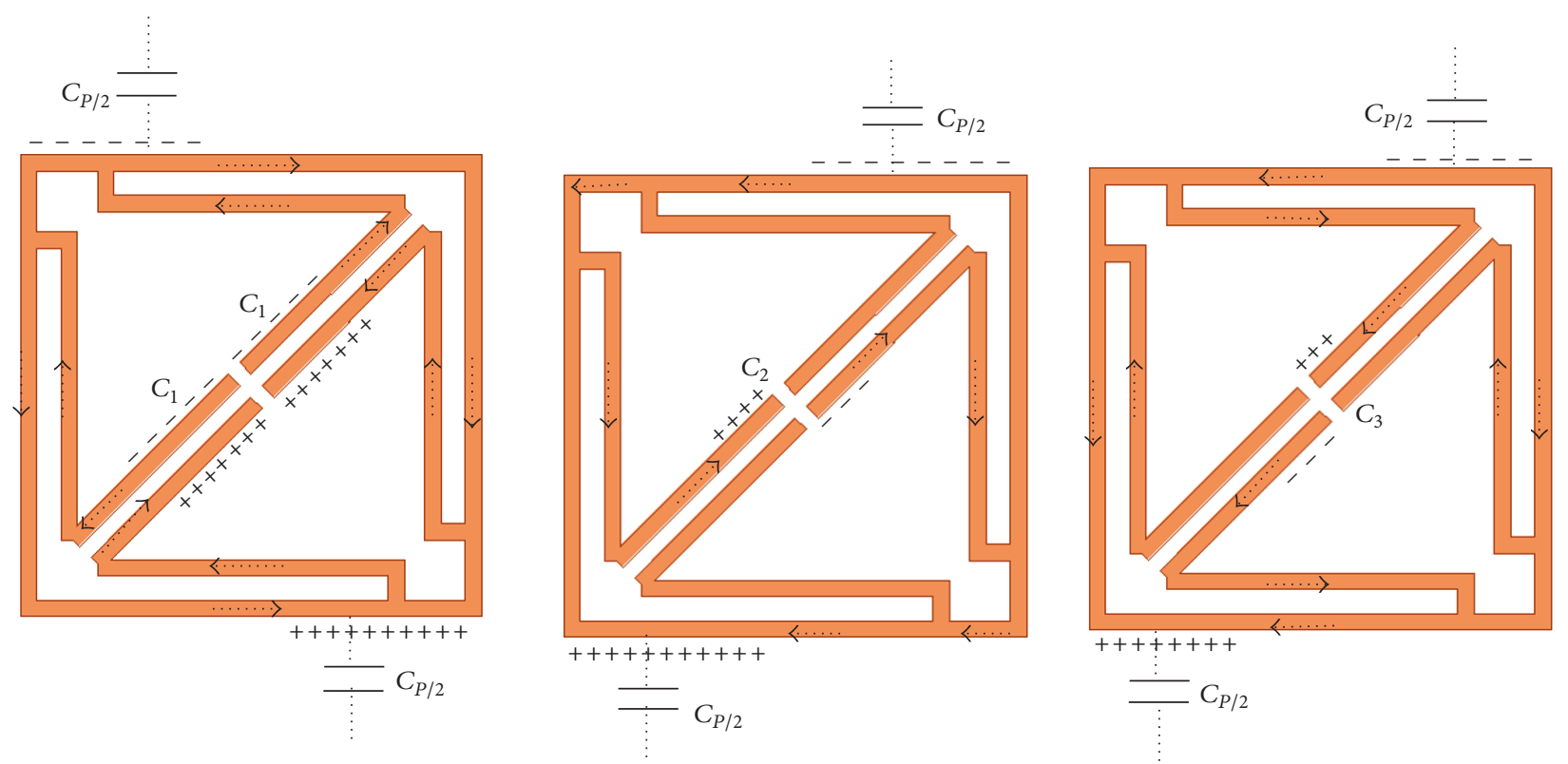

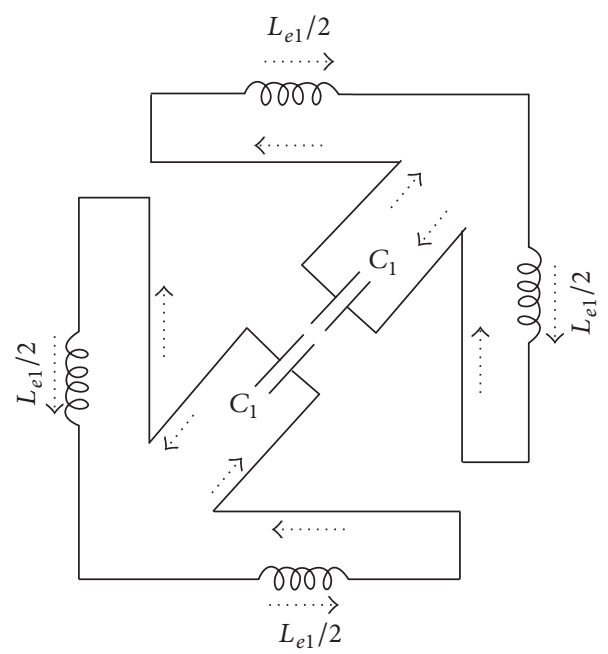

(a)

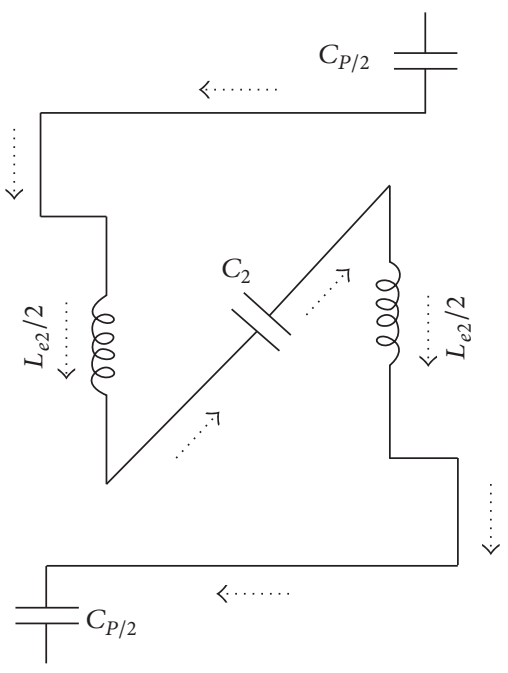

(b)

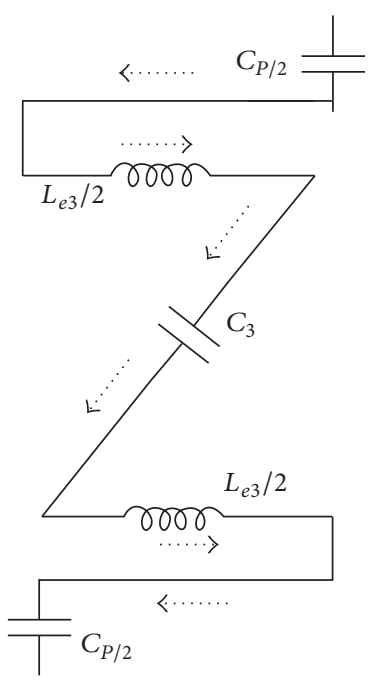

(c)

FIGURE 7: Equivalent circuit topology for geometry of TBEMR at resonant frequencies (a) $f_{01}=4.32 \mathrm{GHz}$, (b) $f_{02}=7.55 \mathrm{GHz}$, and (c) $f_{03}=9.76 \mathrm{GHz}$. Current flow is represented by dashed arrow and capacitances are denoted by $C_{1}, C_{2}, C_{3}$, and $C_{p}$.

$C_{e 2}=C_{p} C_{2} /\left(C_{p}+C_{2}\right)=0.0147 \mathrm{pF}$. The predicted resonant frequency $f_{02}=1 / 2 \pi \sqrt{L_{e 2} C_{e 2}}$ has value $7.51 \mathrm{GHz}$ close to simulated value.

At third resonant mode $f_{03}$, total self-inductance for the current path is $L_{03}=25.5 \mathrm{nH}$ calculated from (5) and mutual inductance $M_{3}$ between the metal strips having antiparallel current has value of $1.5 \mathrm{nH}$ calculated using (6). Then the total inductance is calculated as $L_{e 3}=L_{o 3}-2 M_{3}=$ $22.5 \mathrm{nH}$. Capacitor $C_{3}$ is relatively weaker than $C_{2}$ and it is approximated as $1 / 8$ th that of $C_{1}$. The equivalent capacitance is then calculated as $C_{e 3}=C_{p} C_{3} /\left(C_{p}+C_{3}\right)=0.011 \mathrm{pF}$. The predicted resonant frequency $f_{03}=1 / 2 \pi \sqrt{L_{e 3} C_{e 3}}$ has value 9.8 $\mathrm{GHz}$ close to simulated value. The increased resonant frequency $f_{03}$ as against $f_{02}$ is due to decreased value of inductance $L_{e 3}$ and capacitor $C_{3}$.

\section{Effect of Design Parameters on Resonance Frequency}

Parametric analysis of the TBEMR is carried out in order to point out importance of different geometrical parameters on the LC resonant frequency and guidelines to design such material. Series of numerical simulations are performed concerning variations in the arm length of inner delta loops $\left(l_{s 1}\right.$, $\left.l_{s 2}\right)$, positions of shorting stub $s_{1}, s_{2}\left(l_{x 2}, l_{y 2}\right)$, closing the gaps formed due to arms of open delta loops, metal strip width $w$, 


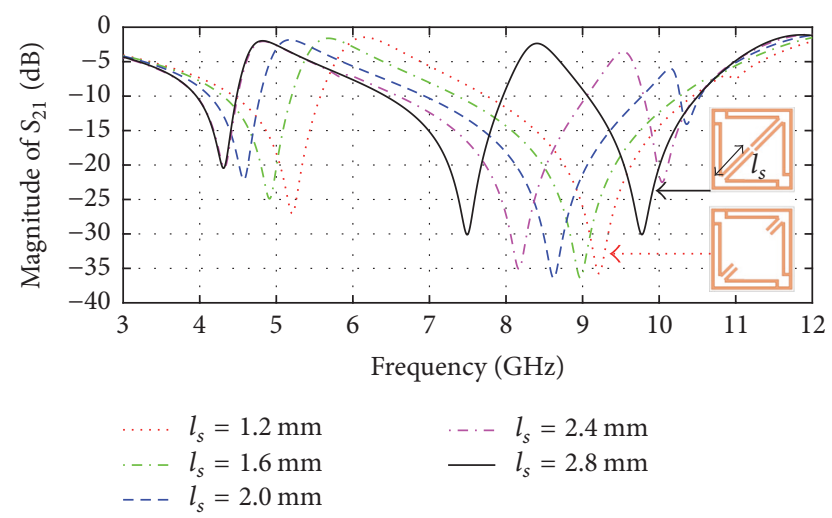

FIGURE 8: Transmission spectra of the TBEMR for different values of arm length $l_{s}$ of the open delta loop.

and substrate properties like permittivity. The transmission spectra under normal incidence are indicted and analyzed for each configuration as presented in subsequent subsection.

4.1. Effect of Changing Arm Length of Inner Delta Loops. The arm length $l_{s}$ for inner delta loops is varied from $l_{s}=$ $1.2 \mathrm{~mm}$ to $l_{s}=2.8 \mathrm{~mm}$ in steps of $0.4 \mathrm{~mm}$. For optimized design as shown in Figure 8, we have considered length $l_{s}=$ $2.8 \mathrm{~mm}$ and three resonances occur at $4.32 \mathrm{GHz}, 7.55 \mathrm{GHz}$, and $9.76 \mathrm{GHz}$, respectively. For decrement in value of $l_{s}$ from optimized value, we find that resonant frequency $f_{01}$ increases to $5.21 \mathrm{GHz}$ (for $l_{s}=1.2 \mathrm{~mm}$ ) from earlier values of $4.32 \mathrm{GHz}$ (for $l_{s}=2.8 \mathrm{~mm}$ ). There is almost a shift of $20 \%$ at resonant frequency $f_{01}$. From simulated surface current density and electric field distribution, it is observed that resonant mode at $f_{01}$ is preserved when arm length $l_{s}$ is decreased. The increase in resonant frequency $f_{01}$ is due to decrease in capacitance $C_{1}$, as $l_{s}$ decreases, whereas second resonant frequency $f_{02}$ shifts upward from value of $7.5 \mathrm{GHz}$ (for $l_{s}=$ $2.8 \mathrm{~mm}$ ) to $8.62 \mathrm{GHz}$ (for $l_{s}=2.04$ ). For further decrements in value of $l_{s}=1.2$, the second and third resonance merge into single value at $9.22 \mathrm{GHz}$. It is found that resonating modes at $f_{02}$ and $f_{03}$ are preserved up to value of $l_{s}=2.0 \mathrm{~mm}$. The shifts in values of $f_{02}$ and $f_{03}$ are probably due to weaker capacitance $C_{2}$ and $C_{3}$. However for lower values of $l_{s}=1.6 \mathrm{~mm}$ and $l_{s}=1.2 \mathrm{~mm}$ modes at $f_{02}$ and $f_{03}$ merge and new resonating mode is formed.

4.2. Effect of Variations in Position of Shorting Stubs $s_{1}, s_{2}$. The lengths $l_{x 2}$ and $l_{y 2}$ of both the ODLRs are varied to see the effect for the change in position of shorting stubs $s_{1}$ and $s_{2}$ as shown in the inset of the Figure 9. Parameters $\left(l_{x 2}\right.$ and $\left.l_{y 2}\right)$ are varied from optimized value of $3.6 \mathrm{~mm}$ to minimum value of $2.0 \mathrm{~mm}$ in steps of $0.4 \mathrm{~mm}$. It is observed that, for optimized value of $3.6 \mathrm{~mm}$, three resonances are obtained as shown in Table 1. For decreased value of $3.2 \mathrm{~mm}$, resonances at $f_{01}$ and $f_{02}$ shift to higher frequencies. The shift at frequency $f_{02}$ is very small compared to shift at $f_{01}$. For lowest value of $2.0 \mathrm{~mm}$ resonance at $f_{03}$ disappears and two closely spaced resonances $f_{01}$ and $f_{02}$ are observed at $5.4 \mathrm{GHz}$ and $7.32 \mathrm{GHz}$, respectively.

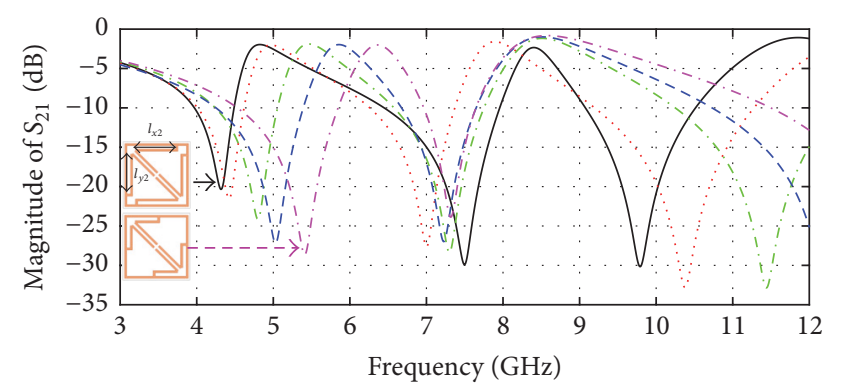

$$
\begin{aligned}
-l_{x 2} & =l_{y 2}=3.6 \mathrm{~mm} \quad \ldots-l_{x 2}=l_{y 2}=2.4 \mathrm{~mm} \\
\ldots \ldots l_{x 2} & =l_{y 2}=3.2 \mathrm{~mm} \\
\cdots-l_{x 2} & =l_{y 2}=2.8 \mathrm{~mm}
\end{aligned}
$$

FIGURE 9: Effect of variations in position of shorting stub $s_{1}, s_{2}\left(l_{x 2}, l_{y 2}\right)$ on $S_{21}$ resonances.

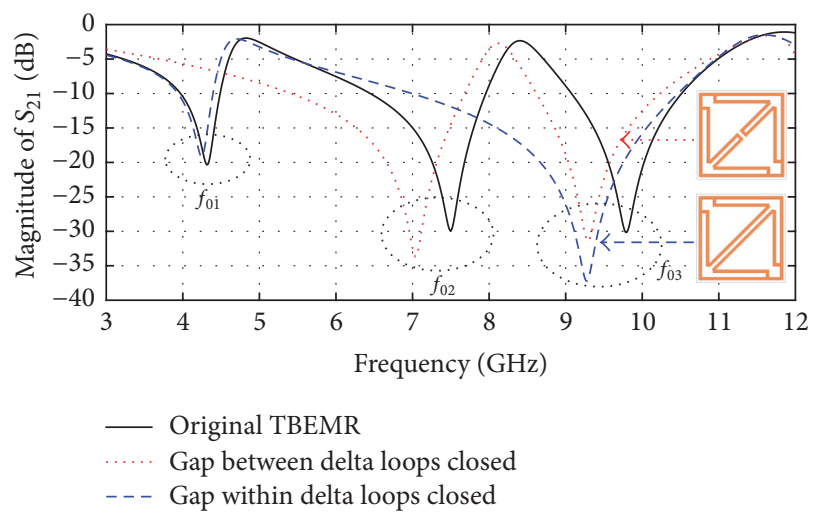

Figure 10: Effect of closed gaps of delta loops on $S_{21}$ resonances.

4.3. Effect of Gaps on Formation of Resonance. To understand the effects of gaps formed due to open delta loops, transmission spectra is computed by closing gaps between and within delta loops as shown in the inset of Figure 10. When gaps between inclined arms $l_{s}$ of open delta loops are closed, it is found that electric resonance at $f_{01}$ totally disappears. This fact confirms the role of capacitance $C_{1}$ in the formation of resonance at $f_{01}$. However, the other two resonances at $f_{02}$ and $f_{03}$ are still present and they are shifted to lower frequencies by $6.13 \%$ and $5.1 \%$. The resonant frequencies at $f_{02}$ and $f_{03}$ are preserved because capacitances $C_{2}$ and $C_{3}$ are preserved as they are formed between diagonally opposite arms $l_{s}$ of the delta loops. These facts are confirmed from the plots of the surface current distributions plotted at resonant frequencies (not shown here). The resonances at $f_{01}$ and $f_{03}$ are present, when gaps within the delta loops are closed in a manner as shown in inset of Figure 10. The resonance at frequency $f_{02}$ disappears totally. The resonance frequency at $f_{01}$ is lowered by $3.33 \%$ and at $f_{03}$ is lowered by $5.1 \%$. These results simply imply that resonant frequencies at $f_{01}$ and $f_{02}$ are directly related to the gaps between and within delta loops, while $f_{03}$ is slightly affected by closing the gaps in either way. The ENG response at first and second band can be tuned by closing corresponding gaps. Moreover, this study also paves 
TABLE 2: Effect of substrate properties on simulated resonant frequency $f_{0}(\mathrm{GHz})$, transmission minima $S_{21}(\mathrm{~dB})$, and effective medium ratio $\left(\lambda_{0} / a\right)$.

\begin{tabular}{lccccc}
\hline Substrate & $\varepsilon_{r}$ & $\tan \delta$ & $f_{0}(\mathrm{GHz})$ & $S_{21}(\mathrm{~dB})$ & $\left(\lambda_{0} / a\right)$ \\
\hline RT Duroid 5880 & 2.2 & 0.0009 & $5.62,9.0,12.07$ & $-33.22,-40.58,-42.41$ & $8.9,5.5,4.14$ \\
FR4 & 4.4 & 0.02 & $4.32,7.55,9.76$ & $-23.29,-34.32,-34.76$ & $11.5,6.6,5.1$ \\
Rogers TMM4 & 4.5 & 0.0020 & $4.36,7.52,9.7$ & $-32.31,-42.16,-39.66$ & $11.46,6.64,5.15$ \\
Alumina & 9.2 & 0.008 & $3.27,5.63,7.22,10.04$ & $-26.43,-35.45,-34.16,-29.16$ & $15.29,8.8,6.9,4.98$ \\
Taconic CER-10 & 10 & 0.0035 & $3.13,5.41,6.94,9.64$ & $-30.86,-39.88,-38,-32.98$ & $15.97,9.24,7.20,5.18$ \\
\hline
\end{tabular}

the way for designing dual and triple-band response and switching the bands at first and second resonance.

4.4. Effect of Changing Substrate Properties. The material properties such as permittivity of the substrate affect resonant frequency and effective medium parameters such as relative permittivity $\varepsilon_{r}$, permeability $\mu_{r}$, and refractive index $n$. To study this effect, we have taken different low and high permittivity substrates like Rogers Duroid $5880 \varepsilon_{r}=2.2$, FR$4 \varepsilon_{r}=4.4$, Rogers TMM4 $\varepsilon_{r}=4.5$, Alumina $\varepsilon_{r}=9.2$, and Taconic CER-10 $\varepsilon_{r}=10$. Considering normal wave incidence setup, it can be found that, without any geometrical and dimensional changes in the structure, a high permittivity substrate presents a lower resonant frequency. The downward shift in resonant frequency can be clearly observed for high permittivity Alumina, Taconic CER-10 substrate as shown in Figure 11. This happens because the capacitance formed between coplanar metallic strips in the structure increases with increase in the permittivity of the substrate, thereby lowering resonant frequency. Another advantage of using high permittivity substrate is that we get improvement in $\lambda_{0} / a$ at respective resonant frequency (refer to Table 2) which is figure of merit for miniaturization and homogenization. We also find that more than three resonances, that is, four, come into picture for high permittivity Alumina and Taconic CER10. Although high permittivity substrates are useful in getting resonant response towards lower frequency, its cost and availability have to be considered. The above study can be employed to optimize a structure for various applications and to get a desired electromagnetic response. Moreover, it is also observed that electric field coupling (dip in $S_{21}$ ) improves for low loss substrate. Low loss Rogers TMM4 has improved coupling compared to that of high loss FR-4 at respective resonant frequencies. Similar observations can be made while comparing Alumina and Taconic CER-10.

4.5. Effect of Changing Metallic Strip Width $w$. For optimized design, we have considered width for metallic strips as $w=$ $0.2 \mathrm{~mm}$. For parametric analysis, width $w$ is varied from $0.18 \mathrm{~mm}$ to $0.28 \mathrm{~mm}$ in steps of $0.02 \mathrm{~mm}$. As seen from plots shown in Figure 12, the variations in resonant frequencies at $f_{01}, f_{02}$, and $f_{03}$ are less than $2 \%, 3.5 \%$, and $4 \%$, respectively. It can be inferred that resonant frequencies do not vary much. However slight increase observed at $f_{02}$ and $f_{03}$ can be attributed to decrease in self-inductance with increment in metallic strip width $w$ [13]. Moreover, it is reported that for very thin metal width conductor losses increase [36].

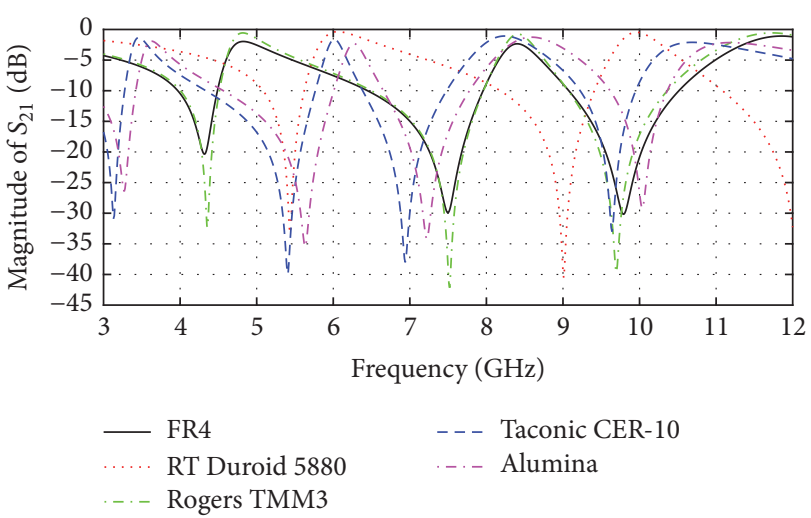

FIGURE 11: Effect of permittivity of the substrate on $S_{21}$ resonance.

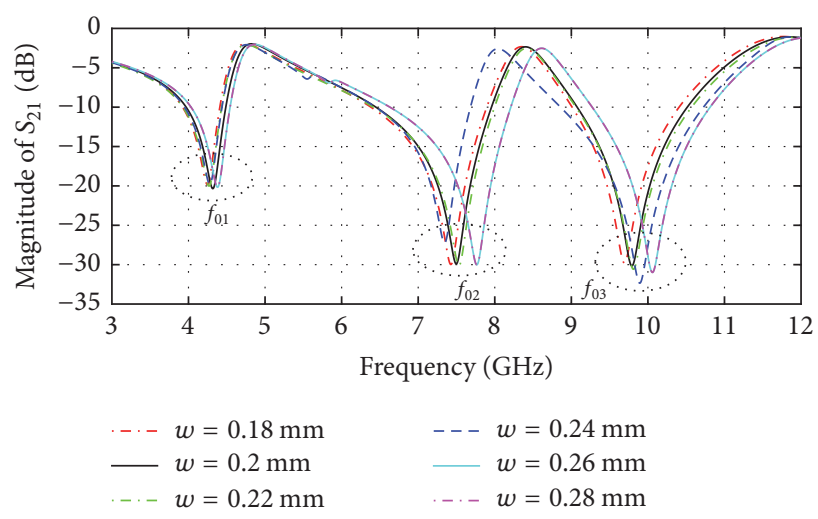

FIGURE 12: Transmission spectra of the TBEMR for different values of metal line width $w$.

Further, performance comparison of the proposed structure with previously reported metamaterial structures is carried out as shown in Table 3. For comparison, we have considered unit cells operating in microwave regime. From Table 3, it is indicated that, compared to $[17,18,20,26]$, the proposed TBEMR structure exhibits triple-band response and highest value of $\left(\lambda_{0} / a\right)$ ratio at lower resonant frequency of $4.32 \mathrm{GHz}$. Moreover at other two resonant frequencies, its value more than 4 is maintained. The proposed structure has advantage of having purely electric response as against $[10,28]$. Letters $\mathrm{E}$ and $\mathrm{M}$ in Table 3 denote type of resonance, namely, electric and magnetic, respectively. An improved $\left(\lambda_{0} / a\right)$ ratio, miniaturization, and triple-band response are the main characteristics of the proposed structure. 
TABLE 3: Performance comparison with reported single negative SNG $(\varepsilon<0$ and/or $\mu<0)$ metamaterial resonators from the literature.

\begin{tabular}{lccccc}
\hline Ref. & Geometry & Cell dimensions $(\mathrm{mm})$ & $f_{0}(\mathrm{GHz})$ & Resonance type & $\left(\lambda_{0} / a\right)$ \\
\hline$[17]$ & E-LC & $3.33 \times 3.33$ & 15.7 & $\mathrm{E}$ & 5.7 \\
{$[18]$} & Idc loaded E-LC & $14 \times 14$ & 2.11 & $\mathrm{E}$ & 10.2 \\
{$[20]$} & Z-shaped & $6 \times 6$ & 4.9 & $\mathrm{E}$ & 10.2 \\
{$[26]$} & Square and meander loaded delta & $7.5 \times 7.5$ & $5.41,6.70$ & $\mathrm{E}, \mathrm{E}$ & $7.39,5.96$ \\
{$[10]$} & Butterfly pattern resonator & $6 \times 8$ & $7.97,10.31,12.08$ & $\mathrm{E}, \mathrm{M}, \mathrm{M}$ & $4.7,3.63,3.10$ \\
{$[13]$} & Nested U-ring resonator & $6.5 \times 6.5$ & $8.24,9.86,12.42$ & $\mathrm{M}, \mathrm{M}, \mathrm{M}$ & $5.6,4.68,3.71$ \\
{$[28]$} & Greek-key pattern & $10 \times 10$ & $2.4,3.5,4$ & $\mathrm{E}, \mathrm{M}, \mathrm{E}$ & $12.5,8.57,7.5$ \\
TBEMR & Square ring, open delta loops & $6 \times 6$ & $4.32,7.55,9.76$ & $\mathrm{E}, \mathrm{E}, \mathrm{E}$ & $11.5,6.6,5.1$ \\
\hline
\end{tabular}

\section{Conclusion}

In conclusion, we present new compact triple-band microwave metamaterials evolved by integrating open delta loops within square ring resonator. The scattering parameters and effective medium parameters of the proposed metamaterial are investigated through full-wave simulation over the frequency range 3 to $12 \mathrm{GHz}$. Proposed geometry has purely electric response as indicated by negative permittivity property at respective resonant frequencies. The effect of various geometrical design parameters on resonant frequencies is investigated and it serves as a guideline to get dual, triple, and tunable response from the proposed structure. Moreover, proposed TBEMR resonator is compact in size as indicated by improved wavelength to unit cell ratio having value of 11.5 and 5.1 at lower and higher resonant frequency, respectively. The proposed metamaterial presents improvement over conventional single-band ENG structures such as thin wire and complementary SRR. The proposed TBEMR is expected to find applications in realization of multiband devices such as antennas, filters, and wave absorbers at microwave and higher frequency regime.

\section{Conflicts of Interest}

The authors declare that there are no conflicts of interest regarding the publication of this paper.

\section{References}

[1] C. Caloz and T. Itoh, Electromagnetic Metamaterials: Transmission Line Theory and Microwave Applications, John Wiley \& Sons, 2005.

[2] D. R. Smith, W. J. Padilla, D. C. Vier, S. C. Nemat-Nasser, and S. Schultz, "Composite medium with simultaneously negative permeability and permittivity," Physical Review Letters, vol. 84, no. 18, pp. 4184-4187, 2000.

[3] R. A. Shelby, D. R. Smith, and S. Schultz, "Experimental verification of a negative index of refraction," Science, vol. 292, no. 5514, pp. 77-79, 2001.

[4] D. Li, Z. Szabo, X. Qing, E.-P. Li, and Z. N. Chen, "A high gain antenna with an optimized metamaterial inspired superstrate," IEEE Transactions on Antennas and Propagation, vol. 60, no. 12, pp. 6018-6023, 2012.

[5] S. Bhattacharyya, S. Ghosh, and K. V. Srivastava, "Bandwidthenhanced metamaterial absorber using electric field-driven
Lc resonator for airborne radar applications," Microwave and Optical Technology Letters, vol. 55, no. 9, pp. 2131-2137, 2013.

[6] M. S. Boybay and O. M. Ramahi, "Material characterization using complementary split-ring resonators," IEEE Transactions on Instrumentation and Measurement, vol. 61, no. 11, pp. 30393046, 2012.

[7] J. Naqui and F. Martín, "Angular displacement and velocity sensors based on electric-LC (ELC) loaded microstrip lines," IEEE Sensors Journal, vol. 14, no. 4, pp. 939-940, 2014.

[8] V. Rawat, V. Nadkarni, and S. N. Kale, "ISM (Industrial Scientific and Medical standard) band flex fuel sensor using electrical metamaterial device," Applied Physics A: Materials Science \& Processing, vol. 123, no. 1, article no. 75, 2017.

[9] O. M. Khan, Z. U. Islam, Q. U. Islam, and F. A. Bhatti, "Multiband high-gain printed yagi array using square spiral ring metamaterial structures for s-band applications," IEEE Antennas and Wireless Propagation Letters, vol.13, pp. 1100-1103, 2014.

[10] G. Du and C. Liu, "Multiband metamaterial structure: Butterflypattern resonator," Microwave and Optical Technology Letters, vol. 54, no. 9, pp. 2179-2181, 2012.

[11] C. Zhu, J.-J. Ma, H.-Q. Zhai, L. Li, and C.-H. Liang, "Characteristics of electrically small spiral resonator metamaterial with electric and magnetic responses," IEEE Antennas and Wireless Propagation Letters, vol. 11, pp. 1580-1583, 2012.

[12] O. Yurduseven, A. E. Yilmaz, and G. Turhan-Sayan, "Hybridshaped single-loop resonator: a four-band metamaterial structure," IEEE Electronics Letters, vol. 47, no. 25, pp. 1381-1382, 2011.

[13] O. Turkmen, E. Ekmekci, and G. Turhan-Sayan, "Nested U-ring resonators: A novel multi-band metamaterial design in microwave region," IET Microwaves, Antennas \& Propagation, vol. 6, no. 10, pp. 1102-1108, 2012.

[14] E. Ekmekci and G. Turhan-Sayan, "Single loop resonator: dualband magnetic metamaterial structure," IEEE Electronics Letters, vol. 46, no. 5, pp. 324-325, 2010.

[15] J. B. Prendry, A. J. Holden, W. J. Stewart, and I. I. Youngs, "Extremely low frequency plasmons in metallic mesostructures," Physical Review Letters, vol. 76, no. 25, pp. 4773-4776, 1996.

[16] F. Falcone, T. Lopetegi, M. A. G. Laso et al., "Babinet principle applied to the design of metasurfaces and metamaterials," Physical Review Letters, vol. 93, no. 19, Article ID 197401, 2004.

[17] D. Schurig, J. J. Mock, and D. R. Smith, "Electric-field-coupled resonators for negative permittivity metamaterials," Applied Physics Letters, vol. 88, no. 4, Article ID 041109, 2006.

[18] W. Withayachumnankul, C. Fumeaux, and D. Abbott, "Compact electric-LC resonators for metamaterials," Optics Express, vol. 18, no. 25, pp. 25912-25921, 2010. 
[19] P. W. Kolb, T. S. Salter, J. A. McGee, H. D. Drew, and W. J. Padilla, "Extreme subwavelength electric GHz metamaterials," Journal of Applied Physics, vol. 110, no. 5, Article ID 054906, 2011.

[20] A. Dhouibi, S. N. Burokur, A. De Lustrac, and A. Priou, "Zshaped meta-atom for negative permittivity metamaterials," Applied Physics A: Materials Science \& Processing, vol. 106, no. 1, pp. 47-51, 2012.

[21] H. Chen, L. Ran, J. Huangfu et al., "Left-handed materials composed of only S-shaped resonators," Physical Review E: Statistical, Nonlinear, and Soft Matter Physics, vol. 70, no. 5, Article ID 057605, 2004.

[22] D. Wang, L. Ran, H. Chen, M. Mu, J. A. Kong, and B.-I. Wu, "Experimental validation of negative refraction of metamaterial composed of single side paired S-ring resonators," Applied Physics Letters, vol. 90, no. 25, Article ID 254103, 2007.

[23] A. Dhouibi, S. N. Burokur, A. De Lustrac, and A. Priou, "Comparison of compact electric-LC resonators for negative permittivity metamaterials," Microwave and Optical Technology Letters, vol. 54, no. 10, pp. 2287-2295, 2012.

[24] Y. Yuan, C. Bingham, T. Tyler et al., "A dual-resonant terahertz metamaterial based on single-particle electric-field-coupled resonators," Applied Physics Letters, vol. 93, no. 19, Article ID 191110, 2008.

[25] A. Sarkhel, D. Mitra, and S. R. Bhadra Chaudhuri, "A compact metamaterial with multi-band negative-index characteristics," Applied Physics A: Materials Science \& Processing, vol. 122, no. 4, pp. 1-10, 2016.

[26] A. Sarkhel, D. Mitra, S. Paul, and S. R. B. Chaudhuri, "A compact meta-atom for dual band negative permittivity metamaterial," Microwave and Optical Technology Letters, vol. 57, no. 5, pp. 1152-1156, 2015.

[27] K. Aydin and E. Ozbay, "Capacitor-loaded split ring resonators as tunable metamaterial components," Journal of Applied Physics, vol. 101, no. 2, Article ID 024911, 2007.

[28] B. Zarghooni, A. Dadgarpour, and T. A. Denidni, "Greek-key pattern as a miniaturized multiband metamaterial unit-cell," IEEE Antennas and Wireless Propagation Letters, vol. 14, pp. 1254-1257, 2015.

[29] B. Li, L. He, Y. Yin, W. Guo, and X. Sun, "An isotropy dual-band terahertz metamaterial," Microwave and Optical Technology Letters, vol. 55, no. 5, pp. 988-990, 2013.

[30] C. Sabah, "Novel, dual-band, single and double negative metamaterials: nonconcentric delta loop resonators," Progress in Electromagnetics Research B, no. 25, pp. 225-239, 2010.

[31] C. Caloz, A. Lai, and T. Itoh, "The challenge of homogenization in metamaterials," New Journal of Physics, vol. 7, article no. 167, 2005.

[32] N. I. Landy, S. Sajuyigbe, J. J. Mock, D. R. Smith, and W. J. Padilla, "Perfect metamaterial absorber," Physical Review Letters, vol. 100, no. 20, Article ID 207402, 2008.

[33] D. R. Smith, D. C. Vier, T. Koschny, and C. M. Soukoulis, "Electromagnetic parameter retrieval from inhomogeneous metamaterials," Physical Review E: Statistical, Nonlinear, and Soft Matter Physics, vol. 71, no. 3, Article ID 036617, 2005.

[34] Z. Szabó, G.-H. Park, R. Hedge, and E.-P. Li, "A unique extraction of metamaterial parameters based on Kramers-Kronig relationship," IEEE Transactions on Microwave Theory and Techniques, vol. 58, no. 10, pp. 2646-2653, 2010.

[35] R. Liu, T. J. Cui, D. Huang, B. Zhao, and D. R. Smith, "Description and explanation of electromagnetic behaviors in artificial metamaterials based on effective medium theory,"
Physical Review E: Statistical, Nonlinear, and Soft Matter Physics, vol. 76, Article ID 026606, 2007.

[36] I. J. Bahl and P. Bhartia, Microwave Solid State Circuit Design, John Wiley \& Sons, 2003.

[37] S. Gevorgian and H. Berg, "Line capacitance and impedance of coplanar-strip waveguides on substrates with multiple dielectric layers," 2001. 


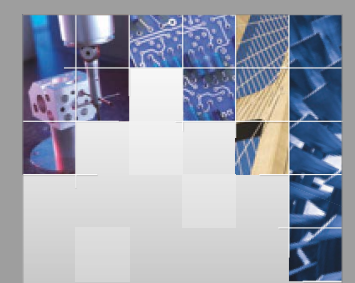

\section{Enfincering}
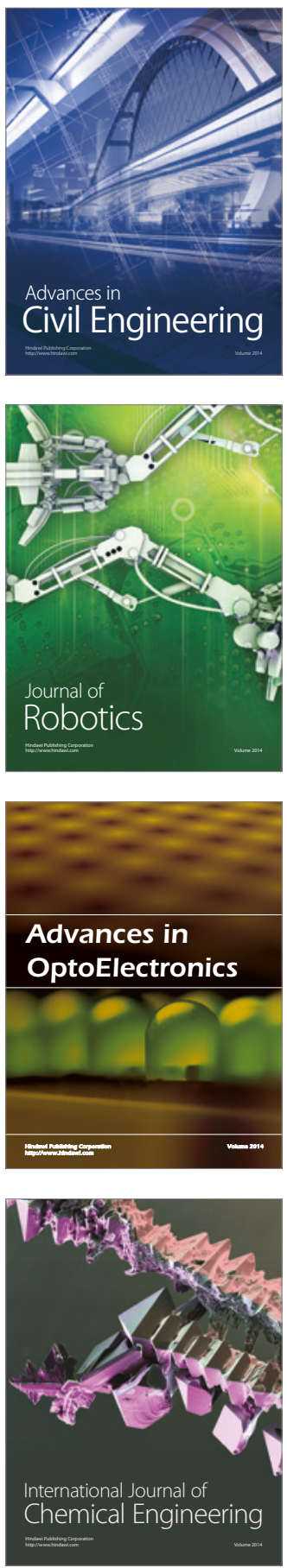

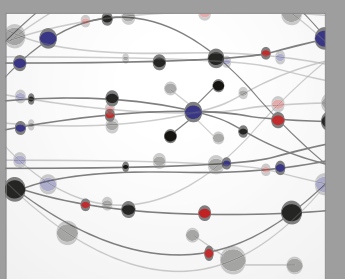

The Scientific World Journal

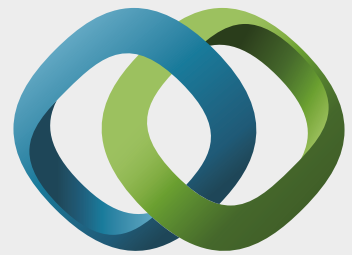

\section{Hindawi}

Submit your manuscripts at

https://www.hindawi.com
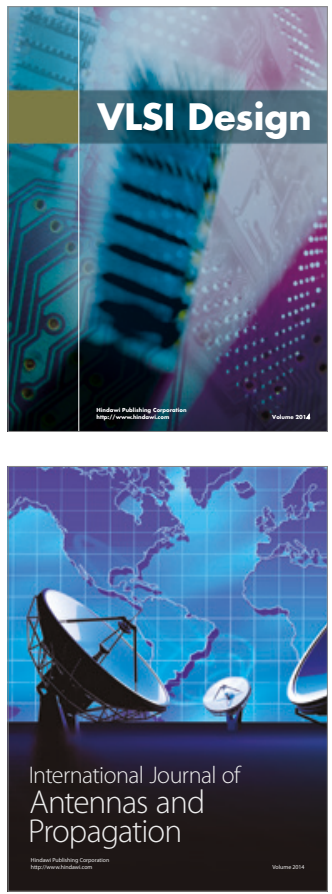

\section{Rotating}

Machinery
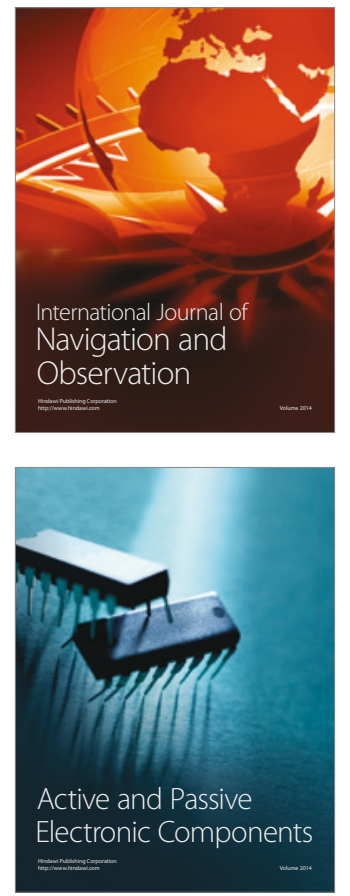
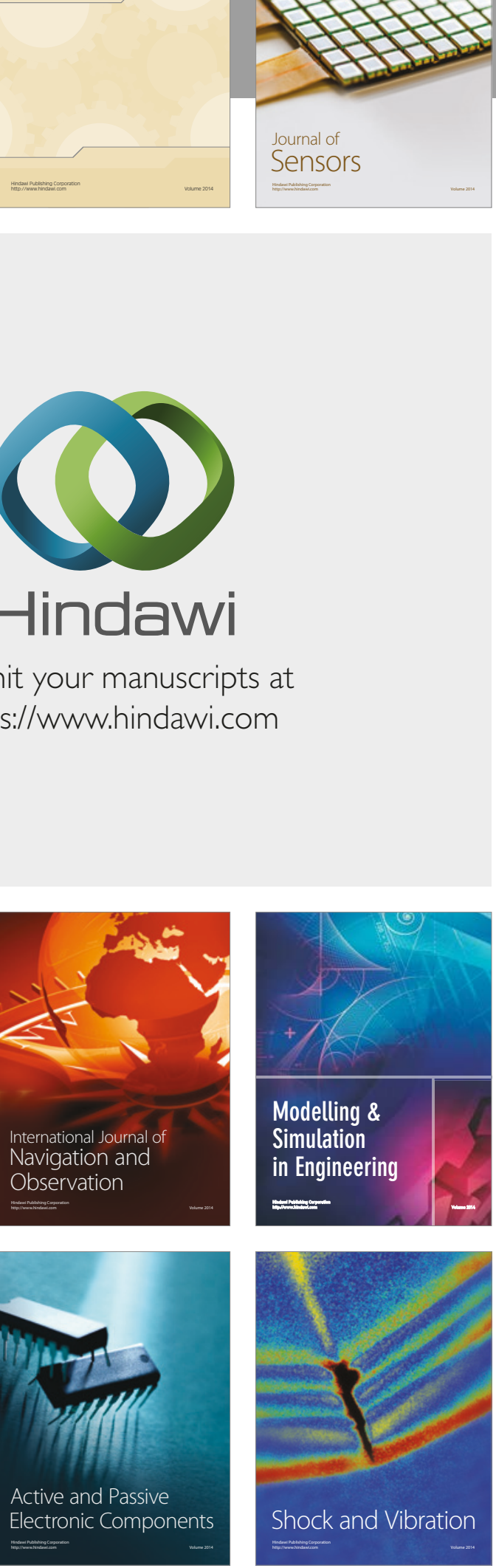
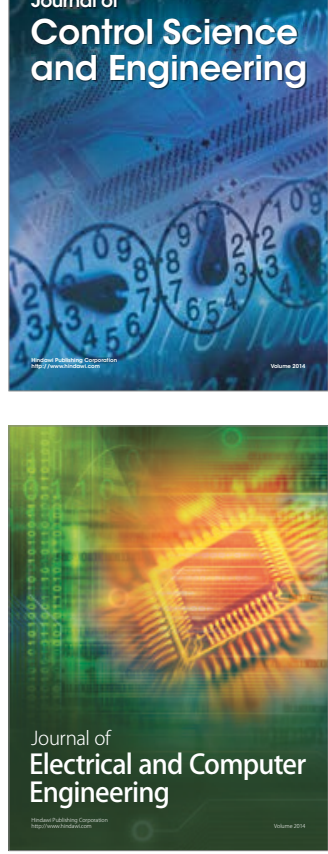

Distributed

Journal of

Control Science

and Engineering
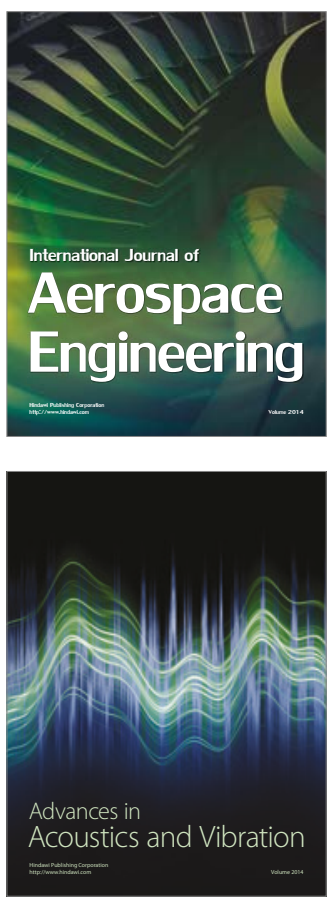

Sensor Networks 